assessment in respect of abnormal behaviour following severe head injury.

His personality type was of the overactive, ambitious, and aggressive type, with an above-average IQ - the epitome of the Type A personality. He ran two businesses simultaneously, and his hobby was competitive cycling. There was no past history or family history of psychiatric illness.

In December 1986 he fell from his push-bike and was found unconscious in the road. He showed signs of a right sub-dural haematoma, and was transferred to a neurosurgical department where CT scan confirmed this. An emergency craniotomy and evacuation of extra-dural and sub-dural clot was performed. At operation, laceration of the right temporal lobe was noted. His post-operative course was stormy - he was unconscious for several days, and then required sedation for restlessness and aggression. He was treated with phenytoin and phenobarbitone. He subsequently made a reasonable recovery physically, with only a persistent right-sided facial nerve palsy and hemianopia.

However, on his return home (three weeks after the injury) he developed markedly abnormal behaviour, with over-activity, outbursts of unprovoked aggression, disinhibition, extreme emotional lability, grandiosity, and irritability. The extent of these symptoms severely threatened his marriage and his business. At this time he refused tranquillisers or psychiatric treatment.

Some weeks later he accepted psychiatric assessment. On admission he presented as an intelligent man, mildly elated in mood, garrulous, and obsessed with his physical fitness. He had the fixed idea that his problems were entirely due to an abnormal glycogen metabolism which he could cure himself by strict dieting. He was physically overactive, but co-operative.

His EEG showed "abnormal asymmetrical activity and a persistent excess of slow activity over the right anterior to mid temporal region". CT brain scan was abnormal, showing "contusion and ischaemia at the right temporal and parietal regions and severe right temporal atrophy". Psychometric testing showed strong evidence of impairment of functioning at the anterior portion of the right temporal lobe (Rey Osterrieth) and also of the frontal lobe (verbal fluency and behaviour on the Wisconsin). It is likely that his frontal lobe pathology is contre coup injury, and the right temporal and parietal signs correlate well with this head injury.

He is now back at work, his mood has improved and stabilised, and he has developed some insight, although he continues to believe that his psychological problems have been due entirely to problems of glycogen metabolism. His wife says he is still "overdoing it", but he is no longer frankly hypomanic.

S. P. MCKEOWN

C. J. JANI

\author{
Altrincham Priory Hospital \\ Rappax Road, \\ Hale, Cheshire WAIS ONX
}

\section{Acute Psychotic Episode Caused by the Abuse of Phensedyl}

SIR: Following the recent publications concerning ephedrine abuse and ephedrine psychosis (Whitehouse \& Duncan, Journal, February 1987, 150, 258-261; Jelley, Journal, September 1987, 151, 418-419), we report a case of phensedyl abuse precipitating an acute psychotic episode.

Case Report: A 49-year-old asthmatic married woman was admitted to our psychiatric unit as an emergency in an agitated and excitable state. She was talking non-stop with some incoherence, was visually hallucinating, said that she was seeing "white spots", and was suspected of hearing voices. She thought that thoughts were being put into her mind and "made her do things", and was disoriented in time, getting the day, month, and year wrong, but was oriented to person and place. She had to be sedated because of her excitability, especially at night, and the psychotic symptoms and disorientation disappeared 48 hours later, at which point the medication was discontinued. Interview 24 hours later confirmed the visual hallucinations and thought insertion, but not the auditory hallucinations. In addition, she described experiencing something like thought broadcasting and passivity feelings. The patient was able to remember most of the period of her delirium with very minor gaps in detail. She stayed in hospital for the next five days and remained symptom-free with no medication.

Her history of phensedyl abuse dated back about 10 years. She remained vague about the amount she consumed, but on checking with her husband it appeared that she had been drinking 3-4 bottles per week. However, the week before her admission she had consumed a larger amount than usual. She also had a history of alcohol abuse, but no other psychiatric history.

\author{
RIADH T. Abed \\ Pamela J. Clark \\ Rotherham District General Hospital \\ Moorgate Road \\ Rotherham S60 2UD
}

\section{Compensation Psychosis}

SIR: In describing a case of compensation psychosis, White et al (Journal, May 1987, 150, 692-694) highlight a topic of growing importance. We report another case of psychosis in the context of compensation, also complicated by bereavement.

Case Report: Our patient was a 36-year-old plumber. Two years previously his wife had died unexpectedly while in hospital, leaving him with three young children. He soon began legal proceedings against the Health Authority concerned, and appeared unable to mourn his wife's death. Fifteen months later his solicitor sought a psychiatric 